\title{
Biomechanical Comparison of Three Kinds of Minimally Invasive Internal Fixation in the Treatment of Pelvic Bilateral Anterior Ring Fractures
}

Yong Zhao ( $\sim$ nihaoedde@126.com)

Yantai Shan Hospital

Yupeng Ma

Yantaishan Hospital

Dexin Zou

Yantaishan Hospital

Xiujiang Sun

Yantaishan Hospital

Gong Cheng

Yantaishan Hospital

Wei Lian

Yantaishan Hospital

Shengjie Dong

Yantaishan Hospital

Yuchi Zhao

Yantaishan Hospital

Wenqing Qu

Yantaishan Hospital

Hao Wu

Yantaishan Hospital

Research article

Keywords: Pelvic anterior ring, Pubic fracture, Minimal invasion, Internal fixation, Biomechanics

Posted Date: May 29th, 2020

DOI: https://doi.org/10.21203/rs.3.rs-30669/v1

License: (c) (i) This work is licensed under a Creative Commons Attribution 4.0 International License.

Read Full License 


\section{Abstract}

[Objective] To compare the mechanical characteristics of percutaneous long plate, percutaneous pubic superioris intramedullary screw and percutaneous pelvic anterior screw-rod system for the treatment of bilateral vertical pubic fractures to provide reference for clinical application.

[Methods] A finite element model of pelvic anterior ring injury (bilateral vertical pubic fracture) was produced. The fractures were fixed with percutaneous long plate, percutaneous pubic superioris intramedullary screw, percutaneous pelvic anterior screw-rod system and their combination in 5 types of models. The fracture stability under vertical, bilateral and anterior-posterior load were quantified and compared based on the displacement of the hip joints' midpoint as quantificational index of fracture stability.

[Results] In condition of bilateral and anterior-posterior load, the vertical, bilateral and anterior-posterior displacement of the hip joints' midpoint of different models were significantly different respectively. In general, the displacement of the 5 pelvic anterior ring fixation models were ranked from maximum to minimum as follows: long plate, pelvic anterior screw-rod system, combination of long plate and pelvic anterior screw-rod system, pubic superioris intramedullary screw and combination of pubic superioris intramedullary screw and pelvic anterior screw-rod system.

[Conclusion] For the fixation in bilateral pubic fractures of pelvic injury, the percutaneous pubic superioris intramedullary screw was optimal, percutaneous pelvic anterior screw-rod system was the second choice, and percutaneous long plate ranked the third. The percutaneous pelvic anterior screw-rod system can significantly increase fixation stability of the percutaneous pubic superioris intramedullary screw and the percutaneous long plate.

\section{Background}

The Tile $B$ and Tile $C$ type pelvic fractures with important organ, blood vessel and nerve injuries are mostly unstable and caused by high energy. In order to save the patients' lives, the final reduction and internal fixation with large iatrogenic injury, more blood loss and long time-consuming cannot be performed in the early stage. Furthermore, due to the special anatomical and physiological characteristics of pelvis, after the improvement of the general situation, the pelvic injury will become old. The difficulty of reduction and fixation will increase significantly and the deformity will be difficult to correct, while the risk of operation such as probability of iatrogenic vascular and nerve injury will also increase significantly. Therefore, in order to reduce the early mortality and disability rate of pelvic injury, it is very important to carry out early, rapid and minimally invasive reduction and internal fixation on the premise that patients can tolerate the treatments.

In order to maximize the stability of pelvis, the combined fixation of anterior and posterior rings is undoubtedly an important means to enhance the effect of internal fixation, which is widely used in unstable pelvic fractures. No matter using the principle of ETC (Early Total Care) to complete the 
reduction and fixation of the two pelvic rings at the same time, or following the principle of DC (Damage Control) to carry out one-stage single ring and two-stage double rings treatment, the anterior ring minimally invasive internal fixation which is not affected by the body position shows the unique advantage. So far, the minimally invasive treatment methods of anterior ring internal fixation mainly include percutaneous bridging long plate (referred to as 'bridging long plate'), percutaneous suprapubic intramedullary screw (referred to as 'intramedullary screw') and percutaneous pelvic anterior ring screwrod system (referred to as 'pelvic anterior ring screw-rod system'). However, the three kinds of anterior ring minimally invasive internal fixation have their own advantages and disadvantages. It is worthy of indepth study that how to choose an effective and safe fixation method according to the specific injury, hardware conditions and technical level, and give full play to the biomechanical advantages of various fixation to achieve the best effect of minimally invasive internal fixation.

Based on this, we use the method of computational biomechanics to simulate the three kinds of minimally invasive internal fixation and their combination to treat the bilateral injury of the anterior ring of pelvis (longitudinal fracture of superior and inferior ramus of pubis) to provide biomechanical support for the optimization of the fixation effect by comparing the biomechanical characteristics and provide biomechanical basis for safe, rapid, minimally invasive, standardized and individualized treatment of such injuries.

\section{Methods}

In this study, the three-dimensional finite element model of pelvis established by our series of research was used, and the related parameters are in line with the relevant literature ${ }^{[1-3]}$. The central sagittal plane of the bilateral superior and inferior ramus of pubis in the normal pelvic model was defined as the fracture surface, and the bilateral longitudinal pubic fracture was simulated.

According to the size of screw, plate and screw rod, a parametric design model was established to simulate the operation mode of percutaneous bridging plate, anterior ring screw-rod system and superior pubic intramedullary screw to fix the superior pubic ramus. The superior pubic intramedullary screw is a cannulated screw with a diameter of $7.3 \mathrm{~mm}$ and a length of $100 \mathrm{~mm}$. The screw is placed retrograde and located in the center of the safe area of the superior ramus of pubis, and the screw thread passes through the fracture line.

The percutaneous bridging plate is a reconstructed plate on the surface of pelvic anterior ring. Its proximal part is located nearby bilateral anterior superior iliac spine and the distal part is located at superior ramus of pubis beside the pubic symphysis. Three cortical screws with $3.5 \mathrm{~mm}$ diameter and 15-32 mm length are placed at the plate proximal and distal ends respectively.

The screw of the anterior ring screw-rod system is $6.5 \mathrm{~mm}$ in diameter and $80 \mathrm{~mm}$ in length. It is inserted from the anterior inferior iliac spine and points to the posterior inferior iliac spine. The pelvic bone and internal fixators were Boolean respectively to form fixation models. 
Simulated fixation modes are as follows: bilateral percutaneous anterior ring bridging plate $(P)$, bilateral percutaneous superior pubic intramedullary screw (S), percutaneous anterior ring screw-rod system (R), combination of bilateral percutaneous anterior ring bridging plate and percutaneous anterior ring screwrod system $(P+R)$, combination of bilateral percutaneous superior pubic intramedullary screw and percutaneous anterior ring screw-rod system (S+R). (Fig. 1-5)

Tie constraints were applied between the bone-implant interfaces except the cannulated screw stem regions where the frictionless sliding contact was applied. Penalty contact with a friction coefficient of 0.3 was applied between the interaction surfaces of fractures. The longitudinal downward load, anteroposterior load and transverse load were simulated to impose to pelvis respectively, and all of them were $600 \mathrm{~N}$.

Under three different loads, the displacement of the virtual bilateral hip midpoints in vertical, transverse and anteroposterior directions were taken as the evaluation index of stability. The smaller is the displacement, the better is the stability.

\section{Results}

In the simulated bipedal standing state, there was no significant difference in the vertical, transverse and anteroposterior displacement of the virtual hip midpoint in the models of bridging plate, superior pubic intramedullary screw, anterior ring screw-rod system and their combined fixation. Similarly, the results of the simulation of standing on one foot were almost same to those of the simulation of standing on two feet mentioned above.

Under the simulated anteroposterior loading condition, there were obvious differences of the virtual hip midpoint's displacement in anteroposterior, transverse and vertical directions in three models of pelvic anterior ring single fixation, in which bridging plate was the largest, and anterior ring screw-rod system was the second, and the intramedullary screw was the smallest. Comparing the models of single and combined fixation, it is shown that the assistant of the anterior ring screw-rod system significantly reduces the displacement of three directions in single bridging plate fixation and sole superior pubic intramedullary screw fixation, and the displacement of the model of superior pubic intramedullary screw combined with anterior ring screw-rod system is the smallest.

Under the simulated left-right loading condition, there were obvious differences of the virtual hip midpoint's displacement in anteroposterior, transverse and vertical directions in three models of pelvic anterior ring single fixation, in which bridging plate was the largest, and anterior ring screw-rod system was the second, and intramedullary screw was the smallest. Comparing the models of single and combined fixation, it is shown that the assistant of the anterior ring screw-rod system significantly reduces the displacement of three directions in single bridging plate fixation and the displacement of leftright and anteroposterior directions in single intramedullary screw fixation, but has no effect on the displacement of vertical direction in single intramedullary screw fixation. The displacement in the model of combination of superior pubic intramedullary screw and anterior ring screw-rod system is the smallest. 
As the models and the loads are bilateral symmetrical, the virtual bilateral hip midpoints' displacements in the same model are not significantly different. Therefore, the pelvic stabilities were compared only with the right hip displacements. (Table. 1-3)

\begin{tabular}{|llll|}
\hline & $\begin{array}{l}\text { Transverse } \\
\text { displacement }\end{array}$ & $\begin{array}{l}\text { Vertical } \\
\text { displacement }\end{array}$ & $\begin{array}{l}\text { Anteroposterior } \\
\text { displacement }\end{array}$ \\
\hline P & 0.06 & 1.14 & 1.03 \\
S & 0.02 & 1.13 & 1.06 \\
\hline R & 0.01 & 1.11 & 1.04 \\
\hline P+R & 0.01 & 1.11 & 1.04 \\
\hline S+R & 0.01 & 1.11 & 1.04 \\
\hline
\end{tabular}

Table 1

The multidirectional displacement under vertical load in the model of simulated standing on two feet $(\mathrm{mm})$

\begin{tabular}{|llll|}
\hline & $\begin{array}{l}\text { Transverse } \\
\text { displacement }\end{array}$ & $\begin{array}{l}\text { Vertical } \\
\text { displacement }\end{array}$ & $\begin{array}{l}\text { Anteroposterior } \\
\text { displacement }\end{array}$ \\
\hline $\mathrm{P}$ & 3.54 & 0.98 & 3.51 \\
$\mathrm{~S}$ & 2.49 & 0.71 & 2.74 \\
$\mathrm{R}$ & 2.80 & 0.89 & 3.05 \\
$\mathrm{P}+\mathrm{R}$ & 2.69 & 0.80 & 2.96 \\
$\mathrm{~S}+\mathrm{R}$ & 2.28 & 0.63 & 2.52 \\
\hline
\end{tabular}

Table 2

The multidirectional displacement under anteroposterior load $(\mathrm{mm})$ 


\begin{tabular}{|llll|}
\hline & $\begin{array}{l}\text { Transverse } \\
\text { displacement }\end{array}$ & $\begin{array}{l}\text { Vertical } \\
\text { displacement }\end{array}$ & $\begin{array}{l}\text { Anteroposterior } \\
\text { displacement }\end{array}$ \\
\hline P & 4.38 & 0.35 & 2.72 \\
\hline S & 0.75 & 0.02 & 0.46 \\
\hline R & 1.53 & 0.25 & 0.98 \\
\hline P+R & 1.37 & 0.16 & 0.85 \\
\hline S $+R$ & 0.60 & 0.02 & 0.37 \\
\hline
\end{tabular}

Table 3

The multidirectional displacement under left-right load $(\mathrm{mm})$

Generally speaking, under simulated anteroposterior and transverse loading conditions, the multidirectional displacement in all models is ranked from small to large as follows: combination of superior pubic intramedullary screw and anterior ring screw-rod system, superior pubic intramedullary screw, combination of bridging plate and anterior ring screw-rod system, anterior ring screw-rod system and pelvic anterior bridging plate. (Table. 1-3)

\section{Discussion}

It is of great therapeutic significance for the injured pelvis with poor stability and multiple complications represented by some Tile $B$ and all Tile $C$ pelvic fractures that how to achieve the effective fixation of the pelvic anterior and posterior rings through minimally invasive treatment, and how to quickly develop the best scheme of minimally invasive fixation according to the time, local conditions and injury conditions.

Progressive and retrograde percutaneous pubic intramedullary screw technique avoids extensive exposure during operation via ilioinguinal approach etc, and achieves ideal clinical results in the treatment of pelvic and acetabular fractures. However, because of the risk of bladder injury, iliac artery and vein injury and hip joint misentry, cannulated screw is a relatively safe choice. Even so, anatomically variant anterior pelvic rings (such as curvature of the superior ramus of pubis, diameter and shape abnormalities of the medullary cavity) often limit the placement of intramedullary screws. Although longer screw can provide better stability, the risk of screw breakage is significantly increased when the screw is too long (length $>100 \mathrm{~mm}$ ) and too thin (diameter $<6.5 \mathrm{~mm}$ ). Therefore, the diameter of superior pubic branch less than $6.5 \mathrm{~mm}$ is also a contraindication of intramedullary screw technique ${ }^{[4]}$. Because the pubis is close to the bladder, iliac artery and iliac vein, retrograde screw placement based on the easily accessible pubic tubercle is safer. With navigation or robot assistance or not, compared with anterograde screw placement, it is more maneuverable and more suitable for one-stage anterior ring internal fixation under the guidance of ETC principle. 
The conventional plate technique of anterior ring combined with posterior ring internal fixation has been used for the final internal fixation of unstable pelvic fracture. However, the technique is not suitable for the early treatment of pelvic injury because the open reduction and internal fixation for anterior ring injury is not minimally invasive. By contrast, anterior ring bridging long plate has the advantage of minimally invasive percutaneous implantation, which effectively avoids the pain of surgical site and incision complications $^{[5]}$, and significantly reduces the incidence of iatrogenic injury such as vascular and nervous system. It can be seen that anterior ring bridging long plate meets various requirements of firststage treatment. Because of the low requirement for the anatomical shape of the anterior pelvic ring, the technique has wide indications and can be used as an effective supplement to the superior pubic intramedullary screw. However, due to the long plate and few screw, its biomechanical properties still need to be further studied.

As an 'inbuilt external fixator', the pelvic anterior screw-rod system creates the principle of fixation above the acetabulum in order to stabilize the anterior pelvic ring by minimal screw and more minimally invasive means, and to help fracture reduction by lateral compression and stretching. It is widely used in Tile $B$ and Tile $C$ pelvic injuries. The fixation is located in the body, does not affect sitting, standing and walking, and is suitable for obese patients especially for severe patients with abdominal organ injury ${ }^{[5]}$. However, the screw placement requires higher technique, and there is a risk of iatrogenic injury of anterolateral femoral cutaneous nerve and hip joint capsule, with a higher incidence of heterotopic ossification ${ }^{[6]}$, and internal fixation needs to be removed by a second operation. In addition, bilateral fixation is necessary for both unilateral and bilateral pubic branch fractures, so the biomechanical characteristics of this fixation and its possible impact on pubic symphysis deserve indepth study.

Thus, three kinds of pelvic anterior ring minimally invasive internal fixation methods have their own advantages and disadvantages. It is worth further study that how to choose an effective and safe fixation method to maximize the biomechanical advantages, avoid internal fixation failure and facilitate early functional exercise.

In this study, it was found that under the simulated bipedal standing state, there were no significant differences in the vertical, bilateral and anteroposterior stability of the fractures between the models of percutaneous superior pubic intramedullary screw, percutaneous bridging plate and percutaneous anterior ring screw-rod system and their combined fixation. Then, the author simulated the state of standing on single foot, and the results were similar to those of the state of standing on two feet. This suggests that the anterior pelvic ring contributes little to the overall vertical stability of the pelvis. Therefore, anterior-posterior loads and right-and-left loads were applied to the pelvis to simulate the mechanism of open-book and lateral damage. By comparing the displacement of the virtual acetabular midpoint in vertical, transverse and anteroposterior directions, the translation and rotation stability of three kinds of anterior ring fixation and their combined fixation on coronal, sagittal and horizontal planes was determined. The results show a significant unity. Comparing the multidirectional stability of three kinds of anterior ring internal fixators' independent fixation, the percutaneous superior pubic intramedullary screw is the best, the percutaneous anterior ring screw-rod system is the second, and the 
percutaneous bridging plate is the worst, which shows the significant mechanical advantage of intramedullary centraxonial fixation. Although the fixation effect of anterior ring screw rod system is affected by indirect fixation across pubic symphysis, it still shows better mechanical characteristics than bridging plate with eccentric fixation. Considering the material consistency of the three internal fixators, it can not be excluded that this result may be related to the bridging plate's 'congenital deficiency' of thin and flat plate and short screws far away from fracture site.

In order to maximize the effect of internal fixation, the author plan to combine three kinds of anterior ring minimally invasive internal fixation devices in pairs. However, the superior pubic intramedullary screw and bridging plate can not be fixed simultaneously because of the limitation of anterior ring anatomy, so only the anterior ring screw-rod system can be combined with the intramedullary screw and bridging plate respectively. The experiment show that, compared with the independent fixation modes, the combined anterior ring internal fixation modes show obvious mechanical advantages, among which the combination of the superior pubic intramedullary screw and anterior ring screw-rod system achieves the most stable fixation effect. Although the combination of bridging plate and anterior ring screw-rod system is superior to their independent fixation, it is still inferior to the single superior pubic intramedullary screw. This suggests that in the clinical practice of fixing bilateral pubic fracture, superior pubic intramedullary fixation should be preferred as far as possible. In order to minimize the failure risk of internal fixation, it is suggested to combine superior pubic intramedullary screw with anterior ring screwrod system fixation. When there is no condition for intramedullary fixation, the combination of anterior ring screw-rod system with bilateral bridging long plate can be used as much as possible. If there is no condition for fixation combination, the anterior ring screw-rod system should be selected as much as possible to avoid the single fixation mode of bilateral long plates.

\section{Conclusions}

For bilateral anterior ring injury of pelvic fracture, in general, there are obvious consistent differences in transverse, vertical and anteroposterior stability of the three internal fixation and their combination; In terms of stability, the combination of percutaneous superior pubic intramedullary screw and percutaneous anterior ring screw-rod system is the best, percutaneous superior pubic intramedullary screw ranks second, percutaneous bridging plate with percutaneous anterior ring screw-rod system is the third, percutaneous anterior ring screw-rod system is the fourth, and percutaneous bridging plate is the worst; Percutaneous anterior ring screw-rod system can significantly increase the stability of percutaneous bridging plate fixation and superior pubic intramedullary screw fixation.

\section{Abbreviations}

1. ' $P$ ' in tables and figure legends is the abbreviation of bilateral percutaneous anterior ring bridging plate.

2. ' $S$ ' in tables and figure legends is the abbreviation of bilateral percutaneous superior pubic intramedullary screw. 
3. ' $R$ ' in tables and figure legends is the abbreviation of percutaneous anterior ring screw-rod system.

4. ' $P+R$ ' in tables and figure legends is the abbreviation of combination of bilateral percutaneous anterior ring bridging plate and percutaneous anterior ring screw-rod system.

5. ' $S+R$ ' in tables and figure legends is the abbreviation of combination of bilateral percutaneous superior pubic intramedullary screw and percutaneous anterior ring screw-rod system.

\section{Declarations}

\section{Ethics approval and consent to participate}

The ethics committees of Yantai Shan Hospital approved the study. The author declares that the methods carried out in this study were in accordance with the relevant guidelines and regulations.

\section{Availability of data and material}

The datasets during and/or analysed during the current study available from the corresponding author on reasonable request.

\section{Competing interests}

The authors declare that they have no competing interests in this section.

\section{Funding}

This research was supported by National Natural Science Foundation of China (No. 81301553 \& No. 81641171), Distinguished Middle-Aged and Young Scientist Encourage and Reward Foundation of Shandong Province, China (No. BS2013SF015), Shandong Provincial Key R\&D Program of China (No. 2018GSF118064), Project of Medical and Health Technology Development Program of Shandong Province, China (No. 2017WS169), Project of Traditional Chinese Medicine Science \& Technology Development Program of Shandong Province, China (No. 2017 - 391) and Project of Science \& Technology Development Program of Yantai City, China (No. 2016WS036). These funding bodies did not participate in the design of the study and collection, analysis, and interpretation of data and writing the manuscript.

\section{Authors' contributions}

Yong Zhao designed and participated in the whole process of the study and drafted the manuscript. Yong Zhao, YM, DZ, XS, GC and WL took part in experimental research. YZ, WQ, HW participated in data collection. SD was involved in data analysis. All authors read and approved the final manuscript. 


\section{References}

1. Zhao Y, Li J, Wang D, et al., (2012) Comparison of stability of two kinds of sacro-iliac screws in the fixation of bilateral sacral fractures in a finite element model. Injury, 43(4): p. 490-494.

2. Zhao Y, Zhang S, Sun T, et al., (2013) Mechanical comparison between lengthened and short sacroiliac screws in sacral fracture fixation: a finite element analysis. Orthop Traumatol Surg Res, 99(5): p. 601-606.

3. Fu S, Zhao Y, Lian W, et al., (2014) Comparison of the risk of breakage of two kinds of sacroiliac screws in the treatment of bilateral sacral fractures. Eur Spine J, 23(7): p. 1558-1567.

4. Suzuki T, Soma K, Shindo M, et al., (2008) Anatomic study for pubic medullary screw insertion. J Orthop Surg, 16(3): p. 321-325.

5. Vaidya R, Colen R, Vigdorchik J, et al., (2012) Treatment of unstable pelvic ring injuries with an internal anterior fixator and posterior fixation: initial clinical series. J Orthop Trauma, 26(1): p. 1-8.

6. Vaidya R, Kubiak EN, Bergin PF, et al., (2012) Complications of anterior subcutaneous internal fixation for unstable pelvis fractures: a multicenter study. Clin Orthop Relat Res, 470(8): p. 21242131.

\section{Figures}

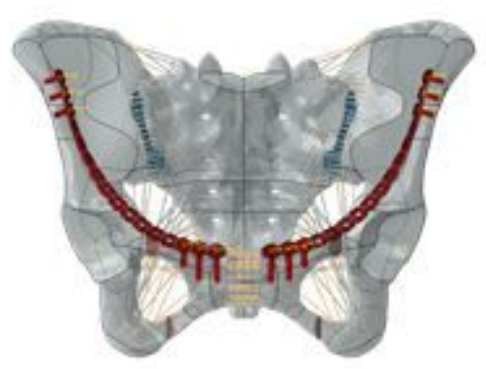

\section{Figure 1}

bilateral percutaneous anterior ring bridging plate $(P)$

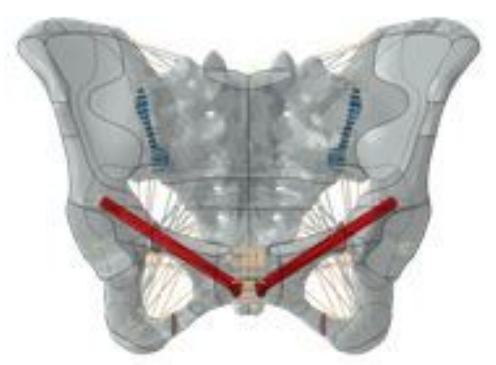

Figure 2 
bilateral percutaneous superior pubic intramedullary screw (S)

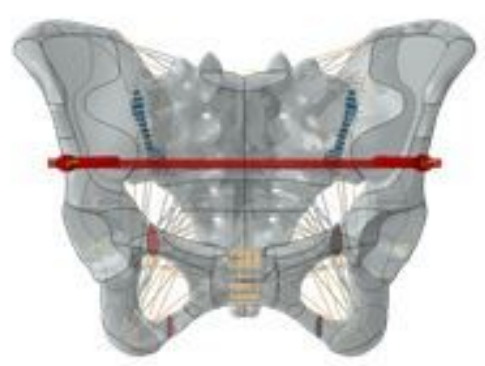

\section{Figure 3}

percutaneous anterior ring screw-rod system (R)

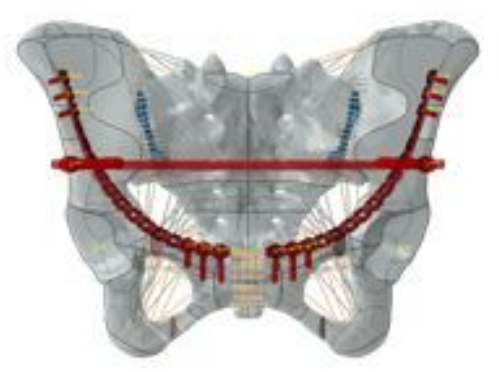

Figure 4

combination of bilateral percutaneous anterior ring bridging plate and percutaneous anterior ring screwrod system $(\mathrm{P}+\mathrm{R})$

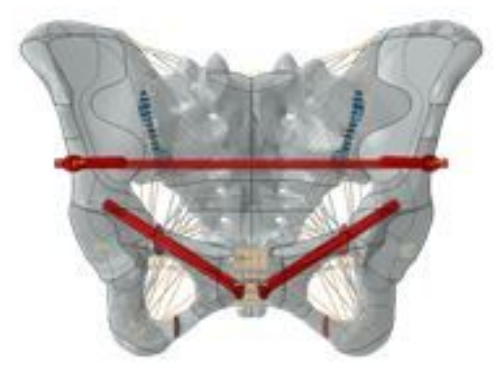

Figure 5

combination of bilateral percutaneous superior pubic intramedullary screw and percutaneous anterior ring screw-rod system $(\mathrm{S}+\mathrm{R})$ 